\title{
Attitude towards working women: Rural-Urban differences (A case study of Pauri town and Buransi village of Uttarakhand)
}

\section{Avritti Purohit ${ }^{1}$ and K.C.Purohit ${ }^{2 *}$}

${ }^{1}$ WPP : Americares India, Haridwar, Uttarakhand

${ }^{2}$ Himalayiy University, Dehradun- 248001

*Corresponding Author Email: kcpisonlinenow@gmail.com

Received: 2.12.2021; Revised: 23.12.2021; Accepted: 28.12.2021

(C) Society for Himalayan Action Research and Development

\begin{abstract}
The role of males and females in different societies has been different because of a number of factors like environmental, economic, social, cultural and political. Even in the developed countries women have not come at par with their male counterparts in terms of status, recognition and active participation in economic activities. However, with the increasing qualitative education, the scenario is changing little by little. In Indian context, in view of multiple societal setup women are still struggling to register their effective presence in the male dominated society in general and poor and backward societies in particular. It is interesting to note that the socio-economic conditions of women in Garhwal region are comparatively better than other regions of the country. Education has played a vital role in bringing about a change in attitude towards women's participation in society. In the present communication an attempt has been made to analyze various factors and indicators associated with the attitude of people towards working women in both urban and rural backgrounds.
\end{abstract}

Keywords: Attitude, Social taboos, Industrial classification, Economic independence

\section{Introduction:}

From time immemorial women throughout the world have been an integral part of the social structure and their role in the society cannot be disguised. The status of women in any civilization shows the stage of evolution of culture in a given time, space and conditions. The attitude towards women in the whole world is variable from country to country based on their cultural values and environment. In England, the entire reform movement centering around women's emancipation had two major aims, firstly, in public life, women should have the same social and political powers as men. Secondly, in private life, women should have parity with men which means women are the competing counterparts (Mill, 1869). In the historical perspective, the role and place of women in the societies has always been fluctuating as per the dictation of social values as well as social taboos. In India in particular, there are sufficient references of high status of 
Himalayan J. Soc. Sci. \& Humanities ISSN: 0975-9891

Vol. 16, (2021) 65-77

DOI: https://doi.org/10.51220/hjssh.v16i1.6

women that underwent drastic change with the Mughal invasion. In the 19th century, however, a number of social reformers made meaningful and serious efforts for the upliftment of women. With the western education the powerful stimulus towards the reform and renovation of women's life came and gripped the contemplating intelligentsia that was steadily beginning to understand its role in the society. The promotion of female education also got impetus. The traditional belief is that man is a social being and he cannot realize himself without the positive assistance of women.

Manu, the great thinker himself has shown his concern for women in a variety of ways. The maxim that 'Women deserve no independence' is not meant by Manu to be taken literally. According to him, within the house, all the members of the family must show the highest regards for the woman. She must be honored and adored by her father, brother and husband who deserve their own welfare (Manusmriti). Kautilya does not hold women in high esteem. He is so suspicious of women that he does not permit a wife even to enter into conversation with any other man except her husband. He also says that marriage is the basis of all disputes (VyavaharaRadha Krishnan, 1973). The Greek philosopher Plato regards women as equal to men and pleads for their emancipation. He says, "Women must have exactly the same education as men." Aristotle was of the opinion that from the hour of their birth some are marked out for the subjection and others for command and declares that women should not be made more like men. According to him, "Silence is woman's glory." (Jowett, 1950)

Status refers to a position in a social system or sub-system which is distinguishable from and at the same time related to other positions through its designated rights and obligations. In the pure sociological sense, status does not imply rank or hierarchy, but denotes only position vis-à-vis others in terms of rights and obligations. The Indian Constitution guarantees equality of opportunities in matters relating to employment and directs the State to secure equal rights to an adequate means of livelihood, equal pay for equal work and just and humane conditions at work (Govt. of India, 1974). Despite all these provisions the status of women in Indian society is still below men and society's attitude towards the working women is still very negative. With the increased educational exposure women in India are being seen in every sphere of industrial classification. In some societies the working of a woman outside home is considered to be prestigious whereas in other societies it is regarded as derogatory. For the economic participation three basic arguments are put forward viz., human rights and social justice, proper utilization of 
human resources and implication of social change.A working woman always faces difficulties in striking balance among her multiple roles. The problem of working women is not only of overburden of duties, but it is also of psychological acceptance of changed circumstances. They are torn between their job and home. They have willingly accepted their dual responsibilities employment and house work. Now, their problem is how to harmonize the two (Myrdal \& Klein, 1968). In Indian background, the ever-present cultural persistence almost in every society with different degrees and in different human groups is the most arresting factor in deciding women's role. The rigidity in the maintenance of systems and customs has resulted in negative impact on women as most of these are only to be followed by females (Purohit \& Zaidi, 1993). It is to be borne in mind that over the last decades there have been marked positive changes in people's perception towards working women due to increasing education and literacy of both males and females.

\section{The Study Area:}

The status and condition of women is relatively better in Garhwal than the rest of India because of a number of geonomic and socio-cultural factors. Due to male outmigration in search of livelihood women in the region have to perform domestic chores, agriculture and job activities whatsoever. This relevant issue has been taken up for the study in Garhwal district of Uttarakhand wherein Pauri town,the headquarters of Garhwal Division and village Buransi of Pabau block have been chosen for the purpose (Fig 1)

Pauri town is situated at $30^{\circ} 8$ ' 9" $\mathrm{N}$ Lat. and $78^{\circ} 9$ ' $8^{\prime \prime}$ E Long. at an elevation of $1650 \mathrm{~m}$ above the MSL. There are 9 wards in the town and 13 regulated/notified villages that make it a municipality. The population as per the 2011 census was 25440 , but presently it is around 40000 persons. It is a multi-religious town sheltering $80 \%$ Hindus, $15.8 \%$ Muslims and 4.2\% Christians. Pauri is functionally an administrative town where there are a large number of employees in more than 150 offices which are mainly occupied by males. The town is characterized by the dominance of tertiary activities followed by primary and secondary activities. The labour sector has been totally dominated by Bihari, Nepali, Bijnauri and Rajasthani workers. 


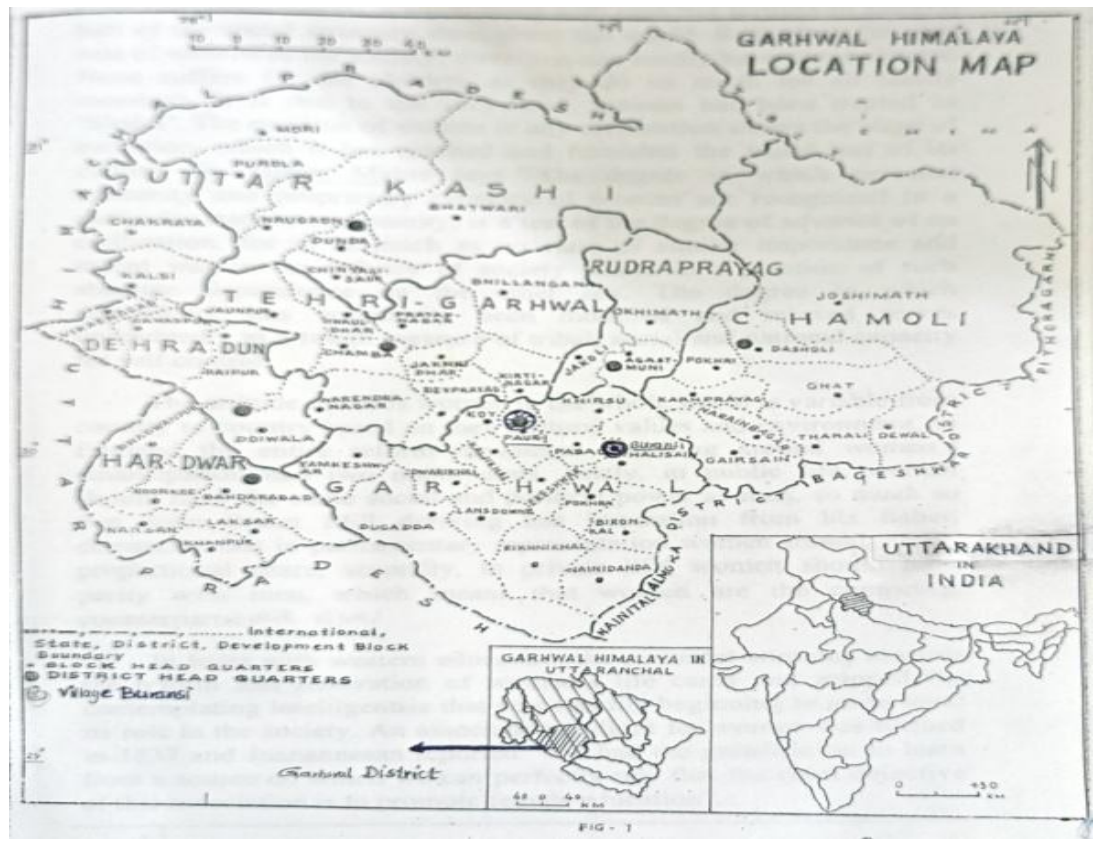

Fig 1: The study Area in District Pauri Garhwal, Uttarakhand

Village Buransi is situated on the Phoolkhal hill of Pabau block at an elevation of $1750 \mathrm{~m}$ at 29 $50^{\prime} 30^{\prime \prime}$ N Lat. and 78 $58^{\prime} 20^{\prime \prime}$ E Long. It is surrounded by Ghuleth and Saknyana villages in the east, Sainji and Gwalkhala in the west, Kolani and Diokot villages in the north while the southern border is demarcated by Deljugad. The topography of the village is rugged and uneven. The population of the village as per the 2011 census was 1200 which has raised upto 1304 in 2017. The sex-ratio is quite high (1213). It is entirely inhabited by Hindus consisting of Rajputs(48\%), SCs(37\%) and Brahmins(15\%).This multi-caste village has relatively low level of literacy. Paithani, Pabau and Majra Mahadev have been serving as good centres of higher education. Being a rural settlement the village has $90 \%$ of its population engaged in agricultural sector and rest $10 \%$ in trade/commerce and higher services.

\section{REVIEW OF LITERATURE}

There are sufficient literary works available on the topic, but in the study area such work is lacking. Radha Dua (2005),Kapoor (1970),Murickan (1975),Shafi (2002),Bowman (1954),Rossi (1964), Talwar (1984), Choi (1987),Tulpule (1977),Pachauri (1994), Mukhopadhyaya (1989), Sharma (1981) and Purohit (1993) have done commendable work related to women, their status, role and economic independence. Nowadays, a number of research projects are being carried out and female-oriented researches are being conducted by regional universities. 
Himalayan J. Soc. Sci. \& Humanities ISSN: 0975-9891

Vol. 16, (2021) 65-77

DOI: https://doi.org/10.51220/hjssh.v16i1.6

\section{AIMS AND OBJECTIVES}

- To assess the status of women in the society of Garhwal both in rural and urban areas

- To analyze the socio-economic and physico-cultural factors responsible in determining the role of women

- To find out various difficulties faced by working women, to examine the ways whereby the women play their multi-functional roles and to mirror how they make an adjustment between home and office

- To identify the views of people regarding the working women and to assess various factors favoring and disfavoring women employment

- To find out rural-urban differences regarding working women

\section{METHODOLOGY}

The work has been based on collection of primary data acquired through door-to-door survey with the help of a detailed questionnaire. During the field visit people from different religions, castes, age-groups, economic status, occupations, sex and literacy levels were interviewed. 100 families each from Pauri town and Buransi village were chosen for the survey. The collected data were tabulated, computed and interpreted. Table 1 gives a detailed account of the surveyed families of the study area:

Table 1: Details of the Families surveyed in urban (Pauri town) and rural (Buransi village) areas (2017)

\begin{tabular}{|c|c|c|c|c|c|c|c|c|c|c|c|c|c|c|}
\hline & Category & $\widetilde{U}$ & & & 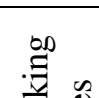 & $\stackrel{\infty}{\Xi} \underset{\square}{\Xi}$ & & ige & oup & the & esp & den & & \\
\hline & & & & & & & $<25$ & & $25-$ & & 40 & & $>5$ & \\
\hline & & & & & & & $\mathrm{M}$ & $\mathrm{F}$ & $M$ & $\mathrm{~F}$ & $\mathrm{M}$ & $\mathrm{F}$ & $\mathrm{M}$ & $\mathrm{F}$ \\
\hline & Hindus & 48 & 21 & 27 & 12 & 14 & 7 & 9 & 7 & 8 & 4 & 7 & 3 & 3 \\
\hline Urban & Brahmins & 15 & 7 & 8 & 5 & 6 & 2 & 2 & 2 & 2 & 2 & 2 & 1 & 2 \\
\hline & Rajputs & 13 & 6 & 7 & 4 & 4 & 2 & 2 & 2 & 2 & 1 & 1 & 1 & 2 \\
\hline & SCs & 20 & 8 & 12 & 3 & 4 & 3 & 3 & 3 & 3 & 1 & 3 & 1 & 3 \\
\hline & Muslims & 32 & 18 & 14 & 9 & 4 & 6 & 3 & 6 & 4 & 4 & 3 & 2 & 4 \\
\hline & Christians & 20 & 10 & 10 & 10 & 5 & 5 & 2 & 4 & 4 & 4 & 2 & 7 & 2 \\
\hline & Total & 100 & 49 & 51 & 27 & 23 & 18 & 14 & 17 & 16 & 12 & 12 & 12 & 9 \\
\hline & Brahmins & 35 & 16 & 19 & 8 & 7 & 4 & 5 & 3 & 4 & 4 & 6 & 5 & 4 \\
\hline Rural & Rajputs & 35 & 18 & 17 & 7 & 4 & 5 & 6 & 4 & 5 & 6 & 3 & 4 & 3 \\
\hline & SCs & 30 & 14 & 16 & 10 & 6 & 3 & 4 & 3 & 3 & 3 & 4 & 5 & 3 \\
\hline & Total & 100 & 48 & 52 & 25 & 17 & 12 & 15 & 10 & 13 & 13 & 13 & 14 & 10 \\
\hline
\end{tabular}

(Source: Field survey) 
Himalayan J. Soc. Sci. \& Humanities ISSN: 0975-9891

Vol. 16, (2021) 65-77

DOI: https://doi.org/10.51220/hjssh.v16i1.6

\section{Exposition}

On the basis of the selection of town and village, their families, heads of families from different age-groups the prepared questionnaire was filled that covered a wide range of questions pertaining to the problem in hand.

\section{Age and Sex-structure of the Population of the families}

The total population of the families in Pauri town was 404 of which 201 were males and 203 females with a sex-ratio of 1010 which was quite higher than the state and national averages of 995 and 940 respectively. As for the village Buransi the sex ratio as expected was 1070 variable from a high of 1829 for SCs to a low of 754 for Brahmins. The total population of the selected families was 526 (Table 2)

Table 2: Age and Sex-structure of the Population of the families (2017)

\begin{tabular}{|c|c|c|c|c|c|c|c|c|c|c|c|c|c|c|c|}
\hline \multirow{3}{*}{ 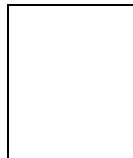 } & \multirow{3}{*}{ Category } & \multicolumn{10}{|c|}{ Age group } & \multirow{2}{*}{\multicolumn{2}{|c|}{ Total }} & \multirow{3}{*}{$\begin{array}{c}\text { Total } \\
\text { populatio } \\
n\end{array}$} & \multirow{3}{*}{$\begin{array}{l}\text { Sex- } \\
\text { ratio }\end{array}$} \\
\hline & & \multicolumn{2}{|c|}{$<5$} & \multicolumn{2}{|c|}{$5-18$} & \multicolumn{2}{|c|}{$19-35$} & \multicolumn{2}{|c|}{$36-60$} & \multicolumn{2}{|c|}{$>60$} & & & & \\
\hline & & $\mathrm{M}$ & $\mathrm{F}$ & $\mathrm{M}$ & $\mathrm{F}$ & $\mathrm{M}$ & $\mathrm{F}$ & $\mathrm{M}$ & $\mathrm{F}$ & M & $\mathrm{F}$ & $\mathrm{M}$ & $\mathrm{F}$ & & \\
\hline & Hindus & 8 & 1 & 1 & 2 & 4 & 3 & 3 & 2 & 7 & 4 & 10 & 95 & 195 & 105 \\
\hline \multirow{7}{*}{$\begin{array}{c}\text { Urba } \\
\mathrm{n}\end{array}$} & & & 3 & 7 & 0 & 5 & 2 & 3 & 6 & & & 0 & & & 2 \\
\hline & Brahmins & 4 & 4 & 3 & 6 & $\begin{array}{l}1 \\
4\end{array}$ & 9 & 9 & 7 & 1 & - & 30 & 26 & 56 & $\begin{array}{c}115 \\
3\end{array}$ \\
\hline & Rajputs & 2 & 7 & 6 & 9 & $\begin{array}{l}1 \\
4\end{array}$ & 8 & 8 & 9 & - & - & 26 & 35 & 61 & 722 \\
\hline & SCs & 2 & 2 & 8 & 7 & $\begin{array}{l}1 \\
7\end{array}$ & $\begin{array}{l}1 \\
6\end{array}$ & $\begin{array}{l}1 \\
6\end{array}$ & $\begin{array}{l}1 \\
0\end{array}$ & 6 & 4 & 44 & 41 & 85 & $\begin{array}{c}107 \\
3\end{array}$ \\
\hline & Muslims & 7 & 9 & $\begin{array}{l}1 \\
7\end{array}$ & $\begin{array}{l}2 \\
1\end{array}$ & $\begin{array}{l}2 \\
3\end{array}$ & $\begin{array}{l}1 \\
9\end{array}$ & $\begin{array}{l}1 \\
9\end{array}$ & $\begin{array}{l}1 \\
9\end{array}$ & 3 & 1 & 61 & 69 & 130 & 884 \\
\hline & $\begin{array}{c}\text { Christian } \\
\text { s }\end{array}$ & 4 & 2 & $\begin{array}{l}1 \\
0\end{array}$ & $\begin{array}{l}1 \\
0\end{array}$ & $\begin{array}{l}1 \\
7\end{array}$ & $\begin{array}{l}1 \\
1\end{array}$ & $\begin{array}{l}1 \\
1\end{array}$ & $\begin{array}{l}1 \\
2\end{array}$ & 2 & 2 & 42 & 37 & 79 & $\begin{array}{c}113 \\
5\end{array}$ \\
\hline & Total & $\begin{array}{l}1 \\
9\end{array}$ & $\begin{array}{l}2 \\
4\end{array}$ & $\begin{array}{l}4 \\
2\end{array}$ & $\begin{array}{l}4 \\
3\end{array}$ & $\begin{array}{l}8 \\
5\end{array}$ & $\begin{array}{l}6 \\
5\end{array}$ & $\begin{array}{l}4 \\
5\end{array}$ & $\begin{array}{l}5 \\
9\end{array}$ & $\begin{array}{l}1 \\
2\end{array}$ & 7 & $\begin{array}{c}20 \\
3\end{array}$ & $\begin{array}{c}20 \\
1\end{array}$ & 404 & $\begin{array}{c}101 \\
0\end{array}$ \\
\hline \multirow{5}{*}{ Rural } & Brahmins & 1 & 1 & 1 & 2 & 2 & 2 & 2 & 2 & 1 & 8 & 77 & 10 & 179 & 754 \\
\hline & Raiputs & $\frac{1}{1}$ & $\frac{2}{2}$ & $\frac{8}{2}$ & $\frac{1}{2}$ & $\frac{7}{2}$ & $\frac{5}{2}$ & $\frac{0}{2}$ & $\frac{6}{2}$ & 1 & 7 & 10 & $\frac{2}{10}$ & 214 & 103 \\
\hline & & 8 & 6 & 8 & 8 & 2 & 2 & 2 & 2 & 9 & & 9 & 5 & & 8 \\
\hline & SCs & 7 & 6 & $\begin{array}{l}2 \\
6\end{array}$ & 2 & $\begin{array}{l}2 \\
6\end{array}$ & 6 & 6 & $\begin{array}{l}2 \\
1\end{array}$ & $\begin{array}{l}2 \\
1\end{array}$ & 1 & 86 & 47 & 133 & $\begin{array}{c}182 \\
9\end{array}$ \\
\hline & Total & $\begin{array}{l}3 \\
6\end{array}$ & $\begin{array}{l}4 \\
4\end{array}$ & $\begin{array}{l}7 \\
2\end{array}$ & $\begin{array}{l}7 \\
0\end{array}$ & $\begin{array}{l}7 \\
5\end{array}$ & $\begin{array}{l}4 \\
8\end{array}$ & $\begin{array}{l}4 \\
8\end{array}$ & $\begin{array}{l}6 \\
9\end{array}$ & $\begin{array}{l}3 \\
2\end{array}$ & $\begin{array}{l}1 \\
6\end{array}$ & $\begin{array}{c}27 \\
2\end{array}$ & $\begin{array}{c}25 \\
4\end{array}$ & 526 & $\begin{array}{c}107 \\
0\end{array}$ \\
\hline
\end{tabular}

(Source: Field survey) 
Himalayan J. Soc. Sci. \& Humanities ISSN: 0975-9891

Vol. 16, (2021) 65-77

DOI: https://doi.org/10.51220/hjssh.v16i1.6

Table 3: Literacy of the respondents

\begin{tabular}{|c|c|c|c|c|c|c|c|c|c|}
\hline \multirow{8}{*}{$\begin{array}{l}\text { Urba } \\
\text { n }\end{array}$} & Category & $\begin{array}{c}\text { Illiterat } \\
\mathbf{e}\end{array}$ & $\begin{array}{c}\text { Primar } \\
\mathbf{y}\end{array}$ & $\begin{array}{c}\mathbf{J H} \\
\mathrm{S}\end{array}$ & $\begin{array}{c}\text { High } \\
\text { Schoo } \\
1 \\
\end{array}$ & $\begin{array}{c}\text { Intermedia } \\
\text { te }\end{array}$ & $\begin{array}{c}\text { Graduatio } \\
\mathbf{n}\end{array}$ & $\begin{array}{l}\mathbf{P} \\
\mathbf{G}\end{array}$ & $\begin{array}{c}\text { Tota } \\
1\end{array}$ \\
\hline & Hindus & 7 & 6 & 5 & 8 & 4 & 12 & 6 & 48 \\
\hline & Brahmins & - & - & 1 & 5 & 1 & 3 & 5 & 15 \\
\hline & Rajputs & - & - & 1 & 3 & 1 & 7 & 1 & 13 \\
\hline & SCs & 7 & 6 & 3 & - & 2 & 2 & - & 20 \\
\hline & Muslims & 9 & 5 & 5 & 6 & 4 & 1 & 2 & 32 \\
\hline & $\begin{array}{c}\text { Christia } \\
\text { ns }\end{array}$ & - & - & 2 & 4 & 7 & 3 & 4 & 20 \\
\hline & Total & 16 & 11 & 12 & 18 & 15 & 16 & 12 & 100 \\
\hline \multirow{4}{*}{ Rural } & Brahmins & 8 & 8 & 7 & 5 & 3 & 4 & - & 35 \\
\hline & Rajputs & 19 & 9 & 3 & 3 & 1 & - & - & 35 \\
\hline & SCs & 22 & 8 & - & - & - & - & - & 30 \\
\hline & Total & 49 & 25 & 10 & 8 & 4 & 4 & - & 100 \\
\hline
\end{tabular}

(Source: Field survey)

\section{Literacy structure:}

The literacy structure of the population is presented in Table 3 which indicated that there were 16 illiterates in the town of which 7 were SCs and 9 were Muslims. Hindus had outnumbered in literacy rate (48). In the village the number of illiterates was 49 , a majority (22) belonged to SC group.

\section{Occupational structure and Income of the families}

The nature of workforce in an area depends upon a variety of economic, demographic, social and environmental factors. In the town only 8 as much as $16 \%$ people were engaged in agricultural sector. A majority of people (37) were found in service of which 26 were Hindus followed by Christians (11). Trade and commerce embraced 44persons of which 29 were Muslims. In the village agriculture formed the prime sector of economy engaging 71 persons. Service sector included 36 persons and other areas had 33 people. The total workforce available in the urban and rural areas was 26.99 and $20.29 \%$ respectively.

Regarding income of the families only 2 families (SC) had an income of less than Rs.5000 per month. 16 families had a monthly income between Rs. 5000 and Rs. 10000 whereas 73 families had an income of more than Rs. 10000 per month (Table 4) 
Himalayan J. Soc. Sci. \& Humanities ISSN: 0975-9891

Vol. 16, (2021) 65-77

DOI: https://doi.org/10.51220/hjssh.v16i1.6

Table 4: Occupational structure and Income of the families (2017)

\begin{tabular}{|c|c|c|c|c|c|c|c|c|}
\hline \multirow{9}{*}{$\begin{array}{l}\text { Urba } \\
\text { n }\end{array}$} & \multirow[t]{2}{*}{ Category } & \multicolumn{3}{|l|}{ Occupation } & \multicolumn{4}{|c|}{ Monthly Income (Rs.) } \\
\hline & & $\begin{array}{l}\text { Agricultur } \\
\text { e/ } \\
\text { Animal } \\
\text { husbandry }\end{array}$ & Service & $\begin{array}{l}\text { Trade/ } \\
\text { Commerc } \\
\text { e }\end{array}$ & Others & $<5000$ & $\begin{array}{l}5000- \\
10000\end{array}$ & $>10000$ \\
\hline & Hindus & $8(16 \%)$ & $26(52 \%)$ & $10(20 \%)$ & $6(12 \%)$ & $2(8 \%)$ & $13(26 \%)$ & $33(66 \%)$ \\
\hline & Brahmins & - & $13(17.3 \%$ & $3(20 \%)$ & $3(6.6 \%)$ & - & $3(20 \%)$ & $12(86.6 \%$ \\
\hline & Rajputs & - & $6(40 \%)$ & $7(46.6 \%)$ & $1(6.6 \%)$ & - & $2(13.3 \%)$ & $\begin{array}{l}13(86.6 \% \\
\end{array}$ \\
\hline & SCs & $8(40 \%)$ & $8(40 \%)$ & - & $4(20 \%)$ & $2(10 \%)$ & $9(45 \%)$ & $9(45 \%)$ \\
\hline & Muslims & - & $1(3.3 \%)$ & $29(96.6 \%)$ & - & - & $1(3.3 \%)$ & $\begin{array}{l}29(96.6 \% \\
)\end{array}$ \\
\hline & $\begin{array}{l}\text { Christian } \\
\text { s }\end{array}$ & - & $11(55 \%)$ & $5(25.2 \%)$ & $4(20 \%)$ & - & $1(3.3 \%)$ & $19(95 \%)$ \\
\hline & Total & 8 & 37 & 44 & 11 & 2 & 16 & $73(14 \%)$ \\
\hline Rural & Brahmins & $22(62.8 \%)$ & $\begin{array}{l}18(51.1 \% \\
)^{2}\end{array}$ & $2(5.7 \%)$ & $9(25.2 \%)$ & $4(14.2 \%)$ & $8(22 \%)$ & $23(65 \%)$ \\
\hline & Rajputs & $33(94.2 \%)$ & $13(37.1 \%$ & - & $8(22.8 \%)$ & $12(34.2 \%$ & $6(17.1 \%)$ & $7(48.5 \%)$ \\
\hline & SCs & $16(53.3 \%)$ & $5(16.6 \%)$ & - & $\begin{array}{l}16(53.3 \% \\
\end{array}$ & $3(10 \%)$ & $\begin{array}{l}19(63.3 \% \\
\end{array}$ & $8(26.5 \%)$ \\
\hline & Total & 71 & 36 & 2 & 33 & 19 & 33 & 48 \\
\hline
\end{tabular}

(Source: Field survey)

\section{Views of respondents about working women}

In order to get information about what people think of working women be that a wife, daughter or daughter-in-law various questions were asked to the respondents and the answers were quite interesting as is evident from the tables ahead. It is to be noted that the questionnaire was prepared in view to know the opinions of the people whether they support or oppose the ideas related to the women desirous of pursuing a job. These data were not at all related to women already in job (Table 5)

Table 5: Views about giving support to wife in service

\begin{tabular}{|c|c|c|c|c|c|c|c|c|c|c|c|c|}
\hline \multirow{6}{*}{$\begin{array}{c}\text { Urba } \\
\text { n }\end{array}$} & Category & $\begin{array}{c}\mathbf{Y e} \\
\mathbf{S}\end{array}$ & $\begin{array}{c}\text { Use of } \\
\text { her } \\
\text { educati } \\
\text { on }\end{array}$ & $\begin{array}{c}\text { Raisi } \\
\text { ng } \\
\text { famil } \\
\mathbf{y} \\
\text { incom } \\
\mathbf{e} \\
\end{array}$ & $\begin{array}{c}\text { Her } \\
\text { rig } \\
\text { ht }\end{array}$ & $\begin{array}{c}\text { Othe } \\
\mathbf{r}\end{array}$ & $\begin{array}{l}\mathbf{N} \\
\mathbf{0}\end{array}$ & $\begin{array}{c}\text { Soci } \\
\text { al }\end{array}$ & $\begin{array}{c}\text { Religio } \\
\text { us }\end{array}$ & $\begin{array}{c}\text { Servic } \\
\text { e of } \\
\text { husba } \\
\text { nd }\end{array}$ & $\begin{array}{c}\text { Negligen } \\
\text { ce } \\
\text { towards } \\
\text { househol } \\
\text { d chores }\end{array}$ & $\begin{array}{c}\text { Othe } \\
\mathbf{r}\end{array}$ \\
\hline & Hindus & 14 & 10 & 3 & 1 & - & 7 & 3 & - & - & 2 & 2 \\
\hline & $\begin{array}{l}\text { Brahmin } \\
\mathrm{s}\end{array}$ & 4 & 2 & 1 & 1 & - & 3 & - & - & - & 1 & 2 \\
\hline & Rajputs & 3 & 1 & 1 & 1 & - & 3 & 1 & - & - & 1 & 1 \\
\hline & SCs & 7 & 1 & 2 & - & - & 1 & - & - & - & - & 1 \\
\hline & Muslims & 6 & 2 & 3 & 1 & 4 & $\begin{array}{l}1 \\
2\end{array}$ & 2 & 5 & 2 & 1 & 2 \\
\hline
\end{tabular}


Himalayan J. Soc. Sci. \& Humanities ISSN: 0975-9891

Vol. 16, (2021) 65-77

DOI: https://doi.org/10.51220/hjssh.v16i1.6

\begin{tabular}{|c|l|c|c|c|c|c|c|c|c|c|c|c|}
\hline & $\begin{array}{l}\text { Christia } \\
\text { ns }\end{array}$ & 8 & 5 & 2 & 1 & - & 2 & - & - & - & 1 & 1 \\
\cline { 2 - 13 } & Total & $\mathbf{2 8}$ & $\mathbf{1 7}$ & $\mathbf{8}$ & $\mathbf{3}$ & $\mathbf{4}$ & $\mathbf{2}$ & $\mathbf{5}$ & $\mathbf{5}$ & $\mathbf{2}$ & $\mathbf{4}$ & $\mathbf{5}$ \\
\hline \multirow{2}{*}{$\begin{array}{c}\text { Rur } \\
\text { al }\end{array}$} & $\begin{array}{l}\text { Brahmin } \\
\text { s }\end{array}$ & 12 & 7 & 3 & 1 & 1 & 4 & 1 & - & - & 1 & 2 \\
\cline { 2 - 13 } & Rajputs & 11 & 5 & 3 & 1 & 2 & 7 & 2 & - & 1 & 3 & 1 \\
\cline { 2 - 12 } & SCs & 11 & 6 & 2 & 2 & 1 & 3 & - & - & - & 2 & 1 \\
\cline { 2 - 12 } & Total & $\mathbf{3 4}$ & $\mathbf{1 8}$ & $\mathbf{8}$ & $\mathbf{4}$ & $\mathbf{4}$ & $\begin{array}{c}\mathbf{1} \\
\mathbf{4}\end{array}$ & $\mathbf{3}$ & - & $\mathbf{1}$ & $\mathbf{6}$ & $\mathbf{4}$ \\
\hline
\end{tabular}

(Source: Field survey)

Table 5 clearly shows that in the town $28(58.33 \%)$ males supported their wives if they wished to work of which 14 were Hindus, 6 Muslims and 8 Christians. $35.41 \%$ wanted their wives to use their education for service while $16.66 \%$ supported them for raising their family income. Only $6.25 \%$ took service as a right of women. The number of persons opposing the service of wife was $21(43.75 \%)$ mostly from the Muslim community (25\%). In the village 34\% people favored working women and $18 \%$ wanted them to use their education. There were 4 people in the town and 6 in the village who opposed wife's employment as they thought it would affect household work.

It is evident from Table 6 that $75 \%$ people from Pauri town supported the employment of their daughters of which $93.75 \%$ were Hindus. Christians also showed positive view in this regard.68\% of the respondents wanted their daughters to use their education, $12 \%$ wanted their economic independence and $8 \%$ supported their working in order to raise their family income. The Muslims (62.5\%) did not support it at all. In the village $85 \%$ people favored working daughters of which $85.71 \%$ were Brahmins.

Table 6: Views about giving support to daughter in service

\begin{tabular}{|c|c|c|c|c|c|c|c|c|c|c|c|c|c|}
\hline & Category & $\begin{array}{c}\text { Ye } \\
\text { s }\end{array}$ & $\begin{array}{c}\begin{array}{c}\text { Use of } \\
\text { her } \\
\text { educati } \\
\text { on }\end{array} \\
\end{array}$ & $\begin{array}{c}\text { Economic } \\
\text { independe } \\
\text { nce }\end{array}$ & $\begin{array}{c}\text { Raisi } \\
\text { ng } \\
\text { famil } \\
\text { y } \\
\text { inco } \\
\text { me }\end{array}$ & $\begin{array}{l}\mathrm{He} \\
\mathbf{r} \\
\text { rig } \\
\text { ht }\end{array}$ & $\begin{array}{c}\text { Oth } \\
\text { er }\end{array}$ & $\begin{array}{l}\mathbf{N} \\
\mathbf{0}\end{array}$ & $\begin{array}{c}\text { Soci } \\
\text { al }\end{array}$ & $\begin{array}{c}\text { Religio } \\
\text { us }\end{array}$ & $\begin{array}{c}\text { Male } \\
\text { 's } \\
\text { ego }\end{array}$ & $\begin{array}{c}\text { Neglice } \\
\text { nce } \\
\text { towards } \\
\text { househo } \\
\text { ld } \\
\text { chores }\end{array}$ & $\begin{array}{c}\text { Oth } \\
\text { er }\end{array}$ \\
\hline \multirow{4}{*}{$\begin{array}{c}\text { Urb } \\
\text { an }\end{array}$} & Hindus & $\begin{array}{l}4 \\
5\end{array}$ & 37 & 4 & 1 & 1 & 2 & 3 & - & - & - & 1 & 2 \\
\hline & $\begin{array}{c}\text { Brahmi } \\
\text { ns }\end{array}$ & $\begin{array}{l}1 \\
5\end{array}$ & 7 & 5 & 2 & - & 1 & - & - & - & - & - & - \\
\hline & Rajputs & $\begin{array}{l}1 \\
3\end{array}$ & 5 & 4 & 3 & - & 1 & - & - & - & - & - & - \\
\hline & $\mathrm{SCs}$ & $\begin{array}{l}1 \\
7\end{array}$ & 3 & 3 & 2 & 8 & 1 & 3 & - & - & - & 1 & 2 \\
\hline
\end{tabular}


Himalayan J. Soc. Sci. \& Humanities ISSN: 0975-9891

Vol. 16, (2021) 65-77

DOI: https://doi.org/10.51220/hjssh.v16i1.6

\begin{tabular}{|c|c|c|c|c|c|c|c|c|c|c|c|c|c|}
\hline & $\begin{array}{c}\text { Muslim } \\
\text { S }\end{array}$ & $\begin{array}{l}1 \\
2\end{array}$ & 5 & 2 & 2 & 1 & 2 & $\begin{array}{l}2 \\
0\end{array}$ & 2 & 12 & 2 & 2 & 2 \\
\hline & $\begin{array}{c}\text { Christi } \\
\text { ans }\end{array}$ & $\begin{array}{l}1 \\
8\end{array}$ & 9 & 3 & 3 & 1 & 2 & 2 & - & - & - & 1 & 1 \\
\hline & Total & $\begin{array}{l}7 \\
5\end{array}$ & 51 & 9 & 6 & 3 & 6 & $\begin{array}{l}2 \\
5\end{array}$ & 2 & 12 & 2 & 4 & 5 \\
\hline \multirow[t]{4}{*}{$\begin{array}{c}\text { Rur } \\
\text { al }\end{array}$} & $\begin{array}{c}\text { Brahmi } \\
\text { ns }\end{array}$ & $\begin{array}{l}3 \\
0 \\
\end{array}$ & 22 & 5 & 2 & - & 1 & 5 & 1 & - & - & 1 & 3 \\
\hline & Rajputs & $\begin{array}{l}2 \\
8 \\
\end{array}$ & 17 & 6 & 3 & - & 2 & 7 & 2 & - & - & 2 & 3 \\
\hline & SCs & $\begin{array}{l}2 \\
7\end{array}$ & 10 & 7 & 3 & 7 & - & 3 & - & - & 1 & 1 & 1 \\
\hline & Total & $\begin{array}{l}8 \\
5\end{array}$ & 49 & 18 & 8 & 7 & 3 & $\begin{array}{l}1 \\
5\end{array}$ & 3 & - & 1 & 4 & 7 \\
\hline
\end{tabular}

(Source: Field survey)

Table 7 depicts abrupt change in the attitude of the respondents when it came to the idea of a working daughter in law. Only 50\% supported the issue and $46 \%$ people thought that education should be utilized.37.5\% Hindus and $84.73 \%$ Muslims opposed the concept. The social factors $(36.61 \%)$ were dominant, but negligence of household work $(42.25 \%)$ was much more decisive factor in the rural side.

Table 7: Views about giving support to daughter-in -law in service

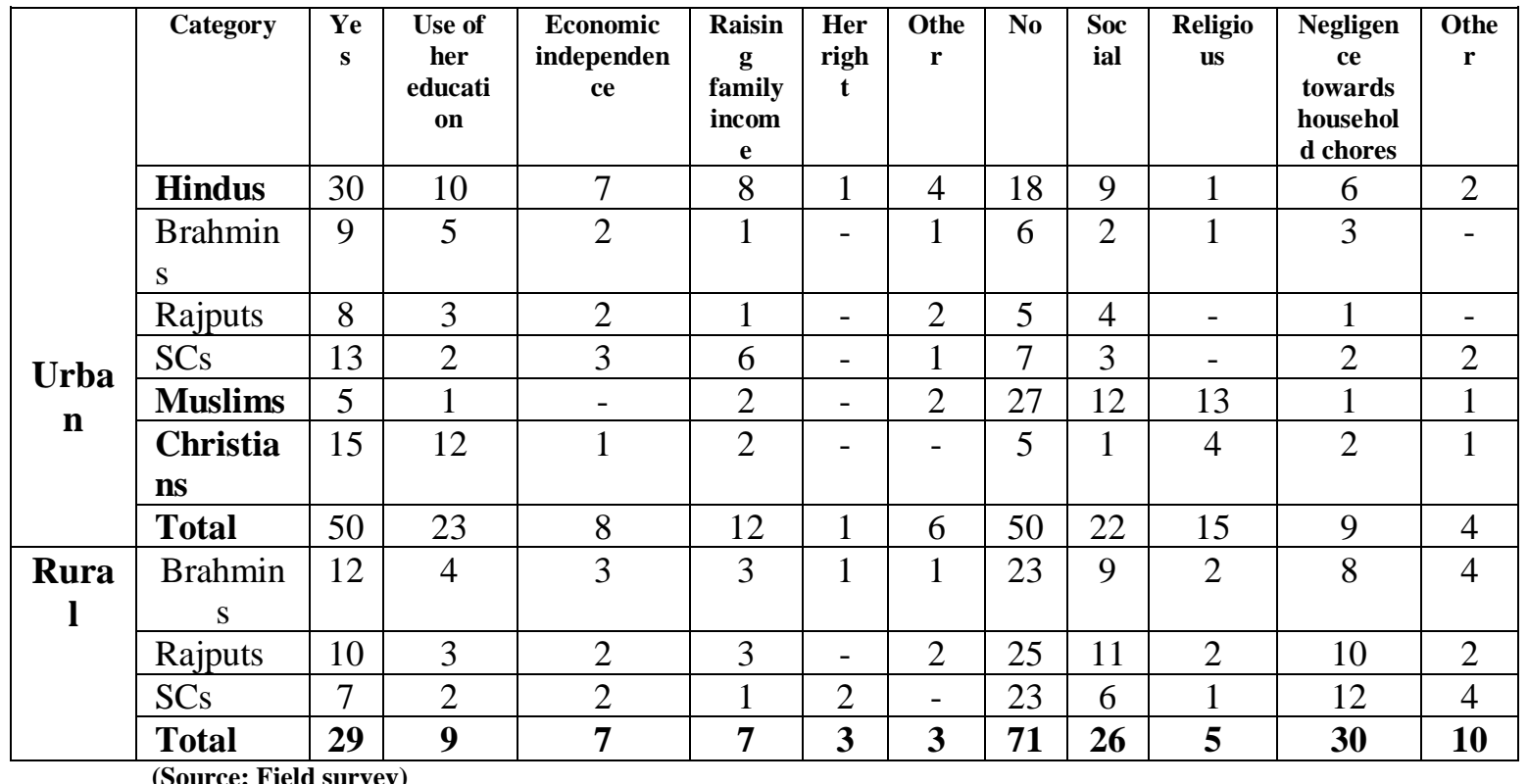


Himalayan J. Soc. Sci. \& Humanities ISSN: 0975-9891

Vol. 16, (2021) 65-77

DOI: https://doi.org/10.51220/hjssh.v16i1.6

Table 8 highlights some of the reasons for supporting and opposing the idea of working women in general. It was a matter of great satisfaction that more than a half of the population both in the urban (56) and rural (68) areas had a positive opinion about working women.

Table 8: Reasons behind supporting and opposing working women

\begin{tabular}{|c|c|c|c|c|c|c|c|c|c|c|c|c|c|c|}
\hline \multirow[t]{8}{*}{ Urban } & 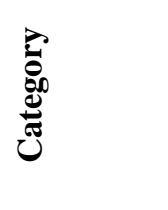 & : & 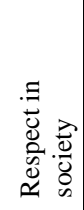 & 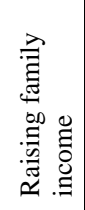 & 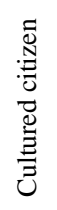 & 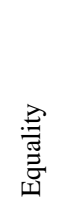 & $\begin{array}{l}\breve{\Xi} \\
\overline{0}\end{array}$ & : & 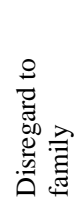 & 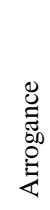 & 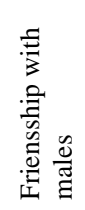 & 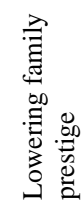 & 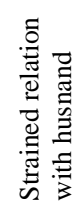 & $\begin{array}{l}\bar{\Xi} \\
\overline{0}\end{array}$ \\
\hline & Hindus & 28 & 7 & 6 & 10 & 5 & - & 14 & 4 & 3 & - & 3 & - & 4 \\
\hline & Brahmins & 8 & 2 & 2 & 3 & 1 & - & 6 & 2 & - & - & 1 & - & 4 \\
\hline & Rajputs & 6 & 2 & 1 & 5 & - & - & 6 & 2 & 1 & - & 1 & - & 2 \\
\hline & SCs & 14 & 3 & 3 & - & - & 8 & 2 & - & - & - & - & - & - \\
\hline & Muslims & 12 & 2 & 6 & 2 & 2 & - & 24 & 2 & 4 & 6 & 6 & - & 4 \\
\hline & Christians & 16 & 5 & 4 & 4 & 1 & 2 & 4 & 2 & - & 1 & - & - & 2 \\
\hline & Total & 56 & 21 & 16 & 24 & 9 & 8 & 42 & 8 & 8 & 7 & 11 & - & 10 \\
\hline \multirow{4}{*}{ Rural } & Brahmins & 24 & 8 & 6 & 3 & 5 & 2 & 12 & 3 & 2 & 1 & - & 1 & 4 \\
\hline & Rajputs & 22 & 7 & 6 & 4 & 2 & 3 & 14 & 6 & 1 & 2 & 1 & 2 & 2 \\
\hline & SCs & 22 & 3 & 4 & 5 & 8 & 2 & 6 & 4 & - & 1 & 1 & - & 2 \\
\hline & Total & 68 & 18 & 16 & 12 & 15 & 7 & 32 & 12 & 1 & 4 & 2 & 3 & 8 \\
\hline
\end{tabular}

In order to identify the difference between working and non-working women some indicators were taken into consideration such as social respect, economic independence, expensive nature, arrogance, etc. $14 \%$ people regarded social respect of a working woman as a differentiating indicator while $12 \%$ supported their economic independence. As regards to their cultured and civilized behavior, $16 \%$ supported them whereas $8 \%$ thought that working women were negligent towards their domestic duties. For the non-working women $23 \%$ people said that these women were economically dependent and $21 \%$ opined that they were responsible towards their family. $17 \%$ thought that these women were content and satisfied. Regarding the rural population the respective figures for responsibility towards home, economic dependence and contentment were 36, 23 and 17\% (Table 9) 
Himalayan J. Soc. Sci. \& Humanities ISSN: 0975-9891

Vol. 16, (2021) 65-77

DOI: https://doi.org/10.51220/hjssh.v16i1.6

Table 9: Difference between working and non-working women

\begin{tabular}{|c|c|c|c|c|c|c|c|c|c|c|c|c|}
\hline & \multirow[t]{2}{*}{ Category } & \multicolumn{6}{|c|}{ Working women } & \multicolumn{5}{|c|}{ Non-working women } \\
\hline & & 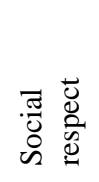 & 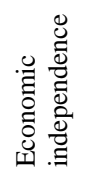 & 总 & 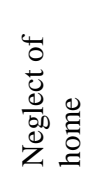 & 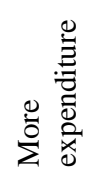 & 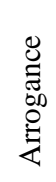 & 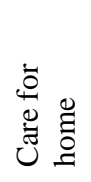 & 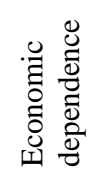 & 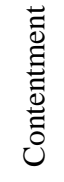 & 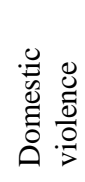 & 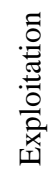 \\
\hline \multirow[t]{7}{*}{ Urban } & Hindus & 7 & 6 & 10 & 4 & 8 & 3 & 8 & 5 & 9 & 6 & 12 \\
\hline & Brahmins & 2 & 2 & 3 & 2 & - & - & 3 & 4 & 2 & 3 & 3 \\
\hline & Rajputs & 2 & 1 & 5 & 2 & - & 1 & 2 & 6 & 3 & 1 & 2 \\
\hline & SCs & 3 & 3 & - & - & 8 & - & 1 & 7 & 2 & 1 & 7 \\
\hline & Muslims & 2 & 6 & 2 & 2 & - & 4 & 10 & 12 & 6 & 2 & 8 \\
\hline & Christians & 5 & 4 & 4 & 2 & 2 & - & 3 & 6 & 2 & 1 & 2 \\
\hline & Total & 14 & 12 & 16 & 8 & 10 & 7 & 21 & 23 & 17 & 9 & 22 \\
\hline \multirow[t]{4}{*}{ Rural } & Brahmins & 8 & 6 & 3 & 3 & 2 & 2 & 12 & 8 & 7 & 6 & 2 \\
\hline & Rajputs & 7 & 6 & 4 & 6 & 3 & 1 & 14 & 9 & 6 & 8 & 4 \\
\hline & SCs & 3 & 4 & 5 & 4 & 2 & - & 10 & 6 & 4 & 5 & 5 \\
\hline & Total & 18 & 16 & 12 & 13 & 7 & 3 & 36 & 23 & 17 & 19 & 11 \\
\hline
\end{tabular}

\section{Conclusion:}

The above analysis makes it clear that the status of women in urban and rural study areas is better than the rest of the country. The geographical conditions have a direct bearing on the overall socio-economic setup and the workforce has shifted from agriculture to secondary and tertiary sectors to a great extent. The views of the people were different in different castes, genders, occupations, age-groups, religions and educational backgrounds both in the town and the village. Highly educated people have a soft corner for women employment. There have been found gaps in the adjustment of jobs and household works. People have been supportive towards the job of their daughters, but opposed to their daughters-in-law if they worked. Most of the people accept that due to the employment of women the economic independence and family income get a rise.

\section{References:}

Bowman,H.A.(1954) : Marriagefor Moderns( $3^{\text {rd }}$ Ed.), McGraw Hill Book Co.,New York

Choi,S.H.L.(1988) : The Marital Adjustment Process of Korean Working class Couples, Ph.D.,Iowa state Univ.,1987,Dissertation Abstracts International ,Vol.49 (2)

Dua, R.(2005) : Working Women, Neelkamal Prakashan,Delhi

Govt. of India (1974) : Report of the Commission on "Status of Women in India",p.266,New Delhi 
Jowett (1950), translation of 'Aristotle's Politics',Oxford University Press,Londo Jowett , translation of 'The Republic', Random House, New York, p.275

Kapoor P.(1970): Marriage and the Working Women in India, Vikas Publishing House,New Delhi Manusmriti, chapter 5,IX,46

Mill JS (1869) : The Subjection of Women, Introduction,Longmans, Green,Reader and Dyer, England,p.12

Mukhopadhyaya S (1989) : Working Status and Stress of Middle class women of Calcutta, Abstracts Women Studies, Vol.7(3)

Murickan J (1975) : Women in Kerala : Changing Socio-Economic Status and Self-Image in Women in Contemporary India, A.Desouza(ed.), Manohar Book Service, Delhi

Myrdal and Klein (1968) : Women's Two Roles - Home and Work, Routledge and Kegan Paul Ltd., London

Pachauri M.L (1974) : A Psychological Study of the family adjustment in relation to self-disclosure among the educated females of Rohilkhand Region of U.P.,Ph.D.Edu.,Rohilkhand University(unpb)

Purohit KC and Zaidi SAH (1993) : Cultural Persistence : A Bottleneck in Women's Develeopmnet, C.M.Agrawal (ed.),Dimensions of Indian Womanhood,Almora, p.299

Purohit,K.C.(1993): Spatial Patterns of Female Workforce in Uttar Pradesh, C.M.Agrawal (ed.), Dimensions of Indian Womanhood,Almora

Radhakrishnan,S. (1973) : Indian Philosophy, Princeton Univ. Press, New Jersey,p.203

Rossi, A. (1964) : 'Equality Between Sexes: An Immodest Proposal', Daedalus 93,p.607-52

Shafi, A. (2002) : Working Women in Kashmir, APH Publishing Corporation, New Delhi

Sharma,M.C.(1981) : A Differential Study of Self-concept,Personality, Adjustment and Values of Teachers at Various Levels, Ph.D. Edu.,Meerut University

Talwar, U. (1984) : Social Profile of Working Women ,Jain Brothers,Jodhpur

Tulpule (1977) : A Pilot study of Adjustment of Pre-University Students of SNDT Arts College for Women,SNDT Uni.,Bombay 